\title{
ACTIVIDAD LIPASA DE ESPECIES DE Malassezia AISLADAS EN PACIENTES SANOS Y CON LESIONES DERMICAS
}

\author{
(Lipase activity of Malassezia species isolated from healthy patients \\ and having dermic lesions)
}

\author{
Maria de los Angeles Sosa, Florencia Rojas, Sergio Toma Vanacore \\ Magdalena Mangiaterra, Gustavo Giusiano \\ Departamento Micología. Instituto de Medicina Regional. \\ Universidad Nacional del Nordeste. Av. Las Heras 727, \\ 3500 Resistencia, Argentina \\ E-mail: gusgiusi@medreg.unne.edu.ar
}

Palabras Claves: Malassezia, activididad lipasa

Key words: Malassezia, lipase activity

\section{RESUMEN}

Las levaduras del género Malassezia forman parte de la microbiota normal de piel humana y animal. Excepto M. pachydermatis, todas las especies de este género son lipodependientes. Bajo ciertos factores, Malassezia se asocia como agente etiológico en diversas afecciones dérmicas. Uno de los principales factores de virulencia de estas levaduras es su actividad de lipasa (AL). El objetivo de este trabajo fue introducir modificaciones a las técnicas de determinación de la actividad lipasa (AL) para su aplicación en levaduras lipodependientes y estudiar la AL en cepas de Malassezia aisladas de personas con piel sana y de pacientes con pitiriasis versicolor $(P V)$, dermatitis seborreica $(D S)$ y psoriasis (PS). Se estudiaron 94 cepas aisladas de 34 pacientes con lesiones de PV, 20 con DS, 7 con PS y 33 cepas de personas con piel sana. Las modificaciones planteadas a la técnica, que incluyeron variación del medio de cultivo y tiempos de incubación, permitieron la determinación semicuantitativa de la AL con resultados claros y definidos. El 88,23\% de las cepas presentó $A L$. No se obtuvieron diferencias estadisticamente significativas al comparar la AL entre las cepas de pacientes con afecciones de piel y las cepas aisladas de personas sanas. La producción de lipasas de las especies de Malassezia en orden decreciente fue: M. sympodialis, M. slooffiae, M. furfur, M. globosa, M. restricta. M. globosa y M. furfur fueron las especies en que se observaron mayor cantidad de cepas no productoras de AL y cepas con gran variabilidad en la medida de $A L$.

Recibido el 23 Abril 2008

Aceptado el 2 Julio 2008

\begin{abstract}
Yeasts of the genus Malassezia are part of the regular microbiota in human and animal skin. Except for M. pachydermatis, all the species of this genus are lipodependent. Malassezia, under certain factors, is associated as an etiological agent in diverse dermic affections. One of the main virulence factors of these yeasts is their lipase activity (LA).

The objective of this research was to introduce some changes in the techniques adopted to determine the lipase activity (LA) in order to apply them to lipodependent yeasts and to study likewise the LA in Malassezia strains isolated from healthy skin people and patients diagnosed with pitiriasis versicolor (VP), greasy dermatitis (GD) and psoriasis (PS). Ninety four strains isolated from 34 patients having VP lesions, 20 with GD, 7 with PS and 33 strains from healthy skin people. Changes suggested to the technique involved a variation in the medium of culture as well as in the time of incubation what resulted in the semiquantitative determination of the LA together with clear and precise results.

The presence of LA was observed in of $88.23 \%$ strains. The comparison of the LA among strains of patients bearing injured skin and those isolated from healthy skin did not show any significant statistical difference. The production of lipasae from Malassezia species were in decreasing order: M.sympodialis, $M$. slooffiae, M.furfur,M.globosa and M.restricta. M. globosa and M. furfur were the species that revealed the highest number of non producting LA strains as well as strains with the highest variability in the degree of $L A$
\end{abstract}




\section{INTRODUCCION}

En contraste con las bacterias, pocos son los hongos que se pueden definir como patógenos primarios y sus mecanismos de patogénesis son mucho menos comprendidos. La mayoría de estos organismos viven como saprófitos ambientales o como simbiontes, coexistiendo con el hospedador sin consecuencias negativas. Sin embargo, algunos son capaces de parasitar hospedadores sanos y generar desde infecciones superficiales hasta las diseminadas o invasoras (1).

Actualmente el género Malassezia está conformado por las siguientes especies: $\boldsymbol{M}$. furfur, $\boldsymbol{M}$. sympodialis, $M$. globosa, M. restricta, M. obtusa, $M$. slooffiae, M. dermatis, M. yamatoensis, M. japonica, M. pachydermatis, M.nana y M. equii. Las 9 primeras forman parte de la microbiota normal de la piel del hombre y las 3 últimas están adaptadas al estrato córneo de los animales $(2,3,4,5,6,7,8)$. Desde el punto de vista fisiológico estas levaduras se distinguen por su lipodependencia, debido a que tienen un defecto en la síntesis de ácidos grasos que se manifiesta en su requerimiento exógeno; excepto $\boldsymbol{M}$. pachydermatis, la cual es lipofílica pero no lipodendiente $(9,10,11,12)$.

Bajo la influencia de ciertos factores, las especies de Malassezia pueden volverse patógenas y participar en diversas enfermedades dermatológicas superficiales e infecciones sistémicas $(2,9,13,14,15)$. Está demostrado que este género es el agente etiológico de la pitiriasis versicolor y la foliculitis y que se asocia a dermatitis seborreica, exacerba la dermatitis atópica y se lo considera como patógeno secundario a otras afecciones de piel. (2, $3,16,17)$.

La virulencia es una compleja interrelación entre el organismo infeccioso y el hospedador. La patogénesis fúngica involucra una interacción y a veces una modificación de factores para ambos $(1,12)$ y uno de los principales en la virulencia de estas levaduras es su actividad de lipasa (17).

El conocimiento sobre los patrones bioquímicos del género Malassezia es exiguo y los escasos informes existentes sobre la producción de lipasa y lipoxigenasa, tanto in vivo como in vitro, fueron realizados antes de la revisión del género cuando se consideraban sólo dos especies, M. furfur y M. pachydermatis $(17,18,19,20)$.

El objetivo de este trabajo fue introducir modificaciones a las técnicas de determinación de la actividad lipasa (AL) para su aplicación en levaduras lipodependientes y estudiar la AL en cepas de Malassezia aisladas de personas con piel sana y de pacientes con pitiriasis versicolor (PV), dermatitis seborreica (DS) y psoriasis (PS).

\section{MATERIALES Y METODOS}

Entre los meses de octubre del 2005 y diciembre de 2007, se estudiaron cepas de Malassezia aisladas de personas que asistieron al Instituto de Medicina Regional (UNNE) y agrupadas de la siguiente manera:

\section{Criterio de inclusión:}

Grupo I: Cepas aisladas de todos los pacientes con PV, DS y PS que demandaron atención.

Grupo II: Cepas aisladas de personas clínicamente sanas, sin lesiones de piel.

\section{Criterios de exclusión:}

Grupo I: Cepas aisladas de pacientes con diagnóstico de PV, DS y PS que no hubieran suspendido el tratamiento tópico u oral 7 días previos a la toma de muestra.

\section{Grupo II:}

- Cepas aisladas de personas clínicamente sanas que presentaran algún antecedente de afección de piel de cualquier índole en los 5 meses precedentes a la toma de la muestra.

- Cepas aisladas de personas con tratamiento antibiótico y/o corticoide durante los 15 días precedentes a la toma de la muestra.

En el caso del Grupo I se tomaron muestras de las lesiones y del Grupo II desde la espalda y el cuero cabelludo. Las muestras fueron recolectadas por raspado con bisturí estéril y sembradas en medio de Agar Dixon modificado e incubadas a $32^{\circ} \mathrm{C}$ hasta 7 días, con observaciones diarias (21)

\section{Identificación}

Todas las cepas de Malassezia aisladas se tipificaron en base a sus características macro y micromorfológicas y a sus propiedades bioquímicas y fisiológicas, según la metodología propuesta por Guillot et al. (21). Para confirmar la identificación se realizaron estudios moleculares, aplicando la metodología de PCRRFLP basada en la técnica de Mirhendi et al. (22).

Como cepas control se incluyeron cepas de referencia CBS 7886 M. globosa, CBS 7019 M. furfur, CBS 7222 M. sympodialis, CBS 9169 M. dermatis, CBS 9432 M. japonica, CBS 10533 M. pachydermatis, CBS 7991 M. restricta, CBS 9725 M. yamatoensis y CBS 7956 M. slooffiae.

\section{Actividad lipasa}

La determinación de la AL se realizó tomando como base la técnica de Riciputo et al. (20), con modificaciones para el estudio de levaduras lipodependientes, introducidas por este grupo de investigación. Al medio base de acuerdo a Riciputo et al. que incluye yema de huevo como fuente de lípidos, se le agregó taurocolato 
Tabla 1: Distribución de especies aisladas según grupo y afección de procedencia

\begin{tabular}{|l|c|c|c|c|c|}
\hline \multirow{2}{*}{ Especie } & \multicolumn{3}{|c|}{ Grupo I } & Grupo II & \multirow{2}{*}{ Total } \\
\cline { 2 - 5 } & $\begin{array}{c}\text { Pitiriasis } \\
\text { Versicolor }\end{array}$ & $\begin{array}{c}\text { Dermatitis } \\
\text { Seborreica }\end{array}$ & Psoriasis & Piel sana & \\
\hline M. furfur & 11 & 5 & 2 & 11 & 29 \\
\hline M. sympodialis & 9 & 3 & 1 & 6 & 19 \\
\hline M. globosa & 14 & 10 & 3 & 14 & 41 \\
\hline M. restricta & 0 & 1 & 1 & 1 & 3 \\
\hline M. slooffiae & 0 & 1 & 0 & 1 & 2 \\
\hline Total & 34 & 20 & 7 & 33 & 94 \\
\hline
\end{tabular}

de sodio como activador de la producción de lipasas. Las placas con medio yema de huevo-taurocolato una vez inoculadas se incubaron a $32^{\circ} \mathrm{C}$. Se hicieron lecturas los días 3, 5, 7 y 10. La formación de zonas de lipólisis o aclaración alrededor de la colonia se consideró indicativa de la producción enzimática.

La medida y el cálculo de la zona de AL se realizó de acuerdo al método descripto por Price et al. (23). La AL se calculó como la relación entre el diámetro de la colonia y el diámetro de la zona de lipólisis $(23,24)$. Estos coeficientes posteriormente se agrupan en 3 clases, lo que permite hacer una medición semicuantitativa de la actividad enzimática:

$$
\mathrm{AL}=\frac{\text { Diámetro de la colonia }}{\text { Diámetro de la zona de producción enzimática }}
$$

AL $<0.69$ : actividad lipasa alta $(+++)$

$\mathbf{0 . 6 9} \geq \mathbf{A L} \leq \mathbf{0 . 8 9}$ : actividad lipasa media $(++)$

$0.9 \geq \mathbf{A L} \leq 1$ : actividad lipasa baja o no detectable $(+)$

Aquellas cepas en las que no se detectó producción de la enzima, es decir, que se obtuvo una $\mathbf{A L}=\mathbf{1}$, se categorizaron como no productoras de lipasa. Para obtener un promedio de AL se midió la AL de 3 colonias de cada cepa estudiada. Todas las determinaciones se realizaron por triplicado.
Se incluyeron cepas de referencia: CBS $7886 \boldsymbol{M}$. globosa, CBS 7019 M. furfur, CBS 7222 M. sympodialis, CBS 7991 M. restricta y CBS 7956 M. slooffiae.

\section{RESULTADOS}

Se estudiaron 94 cepas aisladas de 34 pacientes con lesiones de PV, 20 con DS, 7 con PS y 33 cepas de personas con piel sana. En la Tabla 1 se presenta la distribución de las especies aisladas según el origen de la cepa.

De los 94 aislados de Malassezia spp., 83 (88,23\%) presentaron AL. En la Tabla 2 se muestra el índice de AL por grupo y afección dérmica.

La distribución por especie de los valores de AL de los 94 aislamientos estudiados se muestra en la Tabla 3. El valor mínimo deAL obtenido se ubicó en 0,198 y el máximo en 1 .

La producción de lipasas de las especies de Malassezia en orden decreciente fue: M. sympodialis, $\boldsymbol{M}$. slooffiae, M. furfur, M. globosa, M. restricta.

Respecto a los tiempos de lectura, al 3er día no se observó desarrollo de M. globosa ni de M. restrica. A partir del día 5, todas habían desarrollado e iniciado su actividad. Entre el día 7 y el 10 se observó una disminución en la relación diámetro de la colonia/diámetro de la zona de producción enzimática. Por esta razón se tomó el

\begin{tabular}{|c|c|c|c|c|c|}
\hline & \multirow{2}{*}{$\mathbf{N}$} & \multicolumn{3}{|c|}{ Actividad Lipasa } \\
\hline & & & $\operatorname{cepas} \operatorname{con} \mathrm{AL}$ & $(\mathbf{A L} \pm \mathbf{S D})$ & $\%$ \\
\hline \multirow{3}{*}{ Grupo I } & Pitiriasis versicolor & 34 & 30 & $0,382 \pm 0,246$ & 88.24 \\
\hline & Dermatitis seborreica & 20 & 18 & $0,379 \pm 0,235$ & 85.71 \\
\hline & Psoriasis & 7 & 7 & $0,292 \pm 0,096$ & 100 \\
\hline \multirow[t]{2}{*}{ Grupo II } & Piel sana & 33 & 28 & $0,437 \pm 0,270$ & 84.85 \\
\hline & Total & 94 & 83 & & 88.23 \\
\hline
\end{tabular}

Tabla 2: Actividad lipasa de Malassezia spp. según grupo y cuadro clínico de procedencia.

AL: Índice de actividad lipasa. SD: Desviación estándar 
Tabla 3: Distribución de los valores de AL obtenidos según la especie.

\begin{tabular}{|c|c|c|c|c|c|c|c|c|c|c|c|}
\hline \multirow{3}{*}{ AL } & \multirow{3}{*}{ Clase } & \multicolumn{10}{|c|}{ Especie } \\
\hline & & \multicolumn{2}{|c|}{ M. furfur } & \multicolumn{2}{|c|}{ M. globosa } & \multicolumn{2}{|c|}{ M. sympodialis } & \multicolumn{2}{|c|}{ M. restricta } & \multicolumn{2}{|c|}{ M. slooffiae } \\
\hline & & $\mathrm{n}$ & $\%$ & $\mathrm{n}$ & $\%$ & $\mathrm{n}$ & $\%$ & $\mathrm{n}$ & $\%$ & $\mathrm{n}$ & $\%$ \\
\hline 1,00 & + & 2 & 6.89 & 8 & 19.51 & 0 & 0 & 1 & 33.33 & 0 & 0 \\
\hline $0,90-0,99$ & + & 0 & 0 & 0 & 0 & 0 & 0 & 0 & 0 & 0 & 0 \\
\hline $0,80-0,89$ & ++ & 0 & 0 & 0 & 0 & 0 & 0 & 0 & 0 & 0 & 0 \\
\hline $0,70-0,79$ & ++ & 0 & 0 & 1 & 2.44 & 0 & 0 & 0 & 0 & 0 & 0 \\
\hline $0,60-0,69$ & ++ & 0 & 0 & 0 & 0 & 0 & 0 & 0 & 0 & 0 & 0 \\
\hline $0,50-0,59$ & +++ & 1 & 3.44 & 2 & 4.88 & 0 & 0 & 0 & 0 & 0 & 0 \\
\hline $0,40-0,49$ & +++ & 4 & 13.80 & 4 & 9.75 & 3 & 15.79 & 2 & 66.67 & 0 & 0 \\
\hline $0,30-0,39$ & +++ & 11 & 37.93 & 15 & 36.6 & 2 & 10.53 & 0 & 0 & 2 & 100 \\
\hline $0,20-0,29$ & +++ & 11 & 37.93 & 10 & 24.39 & 9 & 47.37 & 0 & 0 & 0 & 0 \\
\hline \multirow[t]{2}{*}{$0,00-0,19$} & +++ & 0 & 0 & 1 & 2.44 & 5 & 26.31 & 0 & 0 & 0 & 0 \\
\hline & & 29 & 100 & 41 & 100 & 19 & 100 & 3 & 100 & 2 & 100 \\
\hline \multicolumn{12}{|c|}{$*$ Clase +: $0.9 \leq \mathrm{AL} \leq 1$; clase $++: 0.69 \leq \mathrm{Lz} \leq 0.89 ;$ clase $+++: \mathrm{Lz}<0.69$} \\
\hline
\end{tabular}

septimo día como punto de corte para la lectura de AL, cuando las relaciones fueron más altas.

Todos los valores de las Tablas 2 y 3 corresponden a la medida obtenida el día 7. Utilizando la prueba de análisis de la varianza ANOVA para comparar la AL entre las cepas de pacientes con afecciones de piel (Grupo I) y las cepas de personas sanas (Grupo II), no se obtuvieron diferencias estadísticamente significativas.

\section{DISCUSION}

La asociación de las especies de Malassezia a diversas patologías ha impulsado el estudio de su capacidad patogénica. Se considera que la producción de proteinasas y lipasas juega un papel importante en la patogenicidad de estos hongos oportunistas (17). Existe un gran número de publicaciones en las cuales se examina la producción de estas enzimas hidrolíticas en aislados de C. albicans y su papel en la patogénesis de la candidosis invasiva, ya que están relacionadas con su virulencia, pero, existen escasos estudios que investiguen su existencia y su relación con la virulencia de las levaduras del género Malassezia (25-28).

Los pocos trabajos publicados sobre la actividad enzimática de Malassezia fueron realizados antes de la revisión del género, cuando se conocían sólo dos especies, M. furfur y M. pachydermatis $(18,19,20)$. Probablemente esos resultados deben ser reevaluados, ya sea, porque no correspondan a alguna de las especies nombradas, o bien, por la posibilidad de la existencia de mezclas en los cultivos. El amplio espectro de especies de Malassezia reconocido en la actualidad hace interesante el estudio de los patrones bioquímicos y del comportamiento de cada una de ellas.
En este estudio se planteó la utilización de un medio sólido que permite la determinación semicuantitativa de la AL. El agregado de taurocolato dio buenos resultados, ya que los halos de producción enzimática obtenidos siempre fueron claros y definidos, por lo tanto, de fácil lectura y apreciación.

Debido a que el género Malassezia se desarrolla más lentamente en cultivo que otras levaduras, como Candida, el tiempo de incubación fue más prolongado que el de la técnica original que fue ideada para levaduras no lipodependientes (20). Malassezia se desarrolla entre 5 y 7 días, por lo que su mayor actividad se observó al $7 \mathrm{mo}$ día.

En nuestro trabajo encontramos AL en el $88,23 \%$ de los 94 aislamientos estudiados. Neves et al. (29), en un estudio similar al nuestro, hallaron $100 \%$ de AL en 11 aislamientos de Malassezia. Probablemente la discrepancia en el porcentaje de positividad se relacione con el número de cepas estudiado ya que aquellos autores sólo encontraron M. furfur y M. sympodialis.

En nuestro trabajo $\boldsymbol{M}$. sympodialis fue la especie que mostró mayor AL. Por otro lado, las 2 cepas de $\boldsymbol{M}$. slooffiae presentaron alta AL. Dado el bajo número de aislamientos obtenidos, es difícil obtener una conclusión de valor sobre esta especie.

M. globosa y M. furfur fueron las especies en que se observaron, por un lado mayor cantidad de cepas no productoras de actividad, y por otro, cepas con gran variabilidad en cuanto a la medida de AL, como así también, en cuanto a su distribución en relación a las patologías en que se asocian y su presencia en piel sana.

Si bien esta claro que la actividad lipolítica es esencial para el crecimiento de Malassezia, al parecer no es su única función. Los lípidos asociados a la pared celular 
han demostrado estar involucrados en una forma de evasión de la respuesta, ya que inhiben la respuesta proinflamatoria normal y esto provee la explicación de su estado de biota comensal en la piel. Además se cree que estos lípidos tienen importancia en la actividad fagocítica, dificultando su internalización y posterior acción microbicida (30).

Se ha postulado que la AL está directamente relacionada a la virulencia, por lo tanto, a mayor AL mayor virulencia (18). En este trabajo no se encontraron diferencias significativas entre los niveles de AL de cepas de afecciones y los de piel sana. Tampoco el índice ANOVA detectó diferencias significativas entre los resultados de las AL comparando los aislamientos de las diferentes afecciones dérmicas estudiadas. La AL debe ser considerada como uno de los factores de virulencia de estas levaduras, pero no el único factor que influye sobre la patogenicidad.

Esta es una contribución al avance en el estudio de la ecología y del rol patogénico de las especies hoy conocidas del género Malassezia.

\section{AGRADECIMIENTOS}

Agradecemos la asistencia técnica de la Sra. Liliana Alegre en el desarrollo del presente trabajo.

\section{REFERENCIAS}

1.- van Burik, J.-A.H. \& Magee, P.T. (2001). Aspect of fungal pathogenesis in humans. Ann. Rev. Microbiol. 55:743-772

2.-Guèho, E.; Boekhout, T.; Ashbee, H.R.; Guillot, J.; Van Belckum, A.; Faergeman, J. (1998). The role of Malassezia species in the ecology of human skin and as pathogen. Med. Mycol. 36 (supp1):220-229

3. Ashbee, H.R. \& Evans, E.G. (2002). Inmunology of Diseases Associated with Malassezia species. Clin. Microbio. Rew. 21-57

4.Guillot, J.; Guého, E.; Lesourd, M.; Midgley, G.; Chèvrier, G.; Dupont, B. (1996). Identification of Malassezia species. A practical approach. J. Mycol. Med. 6:103-110

5.Guého, E.; Midgley, G.; Guillot, J. (1996). The genus Malassezia with description of four new species. Antonie van Leeuwenhoek 69:337-55

6. Takashi, S.; Takashima, M.; Takako, S.; Hajime, S.; Tetsushi, U.; Ryoji, T.; Hideoki, O. (2002). New yeast species, Malassezia dermatis, isolated from patients with atopic dermatitis. J. Clin. Microbiol. 40:1363-1367

7. Sugita, T.; Takashima, M.; Kodama, M.; Tsuboi, R.; Nishikawa, A. (2003). Description of a new yeast species, Malassezia japonica, and its detection in patients with atopic dermatitis and healthy subjects. J. Clin. Microbiol. 41:4695-4699

8. Sugita, T.; Tajima, M.; Takashima, M.; Armaya, M.; Saito, M.; Tsuboi, R.; Nishikawa, A. (2004). A new yeast, Malassezia yamatoensis, isolated from patient with seborrheic dermatitis, and its distribution in patients and healthy subjects. Microbiol. Immunol. 48:576-583

9. Crespo, E.V. \& Delgado, F. V. (2002). Malassezia species in skin diseases. Curr. Opinión Infect. Dis. 15:133-142

10. Mayser, P.; Pichel, M.; Erdmann, F.; Papavassilis, C.; Schmidt, R. (1998). Different utilization of neutral lipids by Malassezia furfur and Malassezia sympodialis. Med. Mycol. $36: 7-14$

11. Midgley, G. (1989). The diversity of Pityrosporum (Malasezia) yeasts in vivo and in vitro. Mycopathologia 106:143-153

12.Cunningham, A.C.; Leeming, J.P.; Ingham, E.; Gowland, G. (1990). Differentiation of three serovars of Malassezia furfur. J. Appl. Bacteriol. 67:439-446

13. Nakabayashi, A.; Sei, Y.; Guillot, J. (2000). Identification of Malassezia species isolated from patients with seborrhoeic dermatitis, atopic dermatitis, pityriasis versicolor and normal subjects. Med. Mycol. 38:337-341

14. Faegemann, J. (2002). Atopic Dermatitis and Fungi. Clin. Microbiol. Rev. 15:545-563

15. Giusiano, G; Mangiaterra, M.; Garcia Saito, V.; Rojas, V.; Gómez, V.; Díaz, M.C. (2006). Fluconazole and Itraconazole Resistance of Yeasts Isolated from the Bloodstream and Catheters of Hospitalized Pediatric Patients. Chemotherapy 52:254-259

16. Crespo E.V.; Ojeda M.A.; Vera C. A.; Crespo E.A.; Sánchez F.F.; Guèho,E. (1999). Mycology of pityriasis versicolor. J. Mycol. Med. 9:143-148

17. Giusiano, G. (2006). Malassezia: Estado del conocimiento y perspectivas en su estudio. Rev. Arg. Microbiol. 38:41-48

18. Mancianti, F.; Rum, A.; Nardoni, S.; Corazza, M. (2000). Extracellular enzymatic activity of Malassezia spp. Isolates. Mycopathologia 149:131-135

19. Cafarchia, C. \& Otranto, D. (2004). Association between Phospholipase Production by Malassezia pachydermatis and Skin Lesions. J. Clin. Microbiol. 42:4868-4869

20. Riciputo, R.M.; Oliveri, S.; Micali, G.; Sapuppo, A. (1996) Phospholipase activity in Malassezia furfur pathogenic strains. Mycoses 39:233-235

21.Guillot, J.; Guého, E.; Lesourd, M.; Midgley, G.; Chèvrier, G.; Dupont, B. (1996). Identification of Malassezia species. A practical approach. J. Mycol. Med. 6:103-110

22. Mirhendi, H.; Makimura, K.; Zomorodian, K.; Yamada, T.; Sugita, T.; Yamaguchi, H. (2005). A simple PCR-RFLP method for identification and differentiation of 11 Malassezia species. J. Microbiol. Methods 61:281-284

23. Price, M.F.; Wilkinson, I.D.; Gentry, L.O. (1982). Plate method for detection of phospholipase activity in Candida albicans. 23. Sabouraudia 20:7-14

24. Coutihno, S.D. \& Paula, C.R. (2000). Proteinase, phospholipase, hyaluronidase and chondroitin-sulphate production by 
Malassezia pachydermatis. Med. Mycol. 38:73-76

25. Chen, S.; Muller, M.; Zhou, J.; Wright. L.; Sorrel, T. (1997). Phospholipase activity in Cryptococcus neoformans: a new virulence factor? J. Infect. Dis. 175:414-420

26. Vidotto, V.; Sinicco, A.; Di Fraia, D.; Cardaropoli, S.; Ito Kuwa, S. (1996). Phospholipase activity in Cryptococcus neoformans. Mycopathologia 136:119-123

27. Banno, Y.; Yamada, T.; Nozawa, Y. (1985). Secreted phospholipases of the dimorphic fungus, Candida albicans; separation of three enzymes and some biological properties. Sabouraudia 23:47-54
28. Barrett-Bee, K.; Hayes, Y.; Wilson, R.G.; Ryley, J.F (1985). A comparison of phospholipase activity, cellular adherence and pathogenicity of yeasts. J. Gen. Microbiol. 131:1217-1221

29. Neves, R.; Magalhães, C.; Lucas da Silva, M.; SouzaMotta, C., Queiroz, L. (2005). Identification and pathogenicity of Malassezia species isolated from human healthy skin and whit macules. Brazilian J. Microbiol. 36:114-117

30. Brunke, S. \& Hube, B. (2006) MfLIP1, a gene encoding an extracellular lipase of the lipid-dependant fungus Malassezia furfur. Microbiology 152:547-554 